\title{
Development of a Bioluminescent Human Osteosarcoma Model in Humanized NSG Mice: A Pilot Study
}

\author{
YUNMI KO ${ }^{1,2^{*}}$, YEON HO JEONG ${ }^{2 *}$, JIN-HEE SEO $^{3}$ and JUN AH LEE ${ }^{1}$ \\ ${ }^{1}$ Department of Pediatrics, Center for Pediatric Cancer, National Cancer Center, Goyang, Republic of Korea; \\ ${ }^{2}$ Department of Medical Biotechnology, College of Biomedical Science, \\ Kangwon National University, Chuncheon, Republic of Korea; \\ ${ }^{3}$ Laboratory Animal Team, Radiation Medicine Support Center, Division of Fusion Radiology Research, \\ Korea Institute of radiological \& Medical Sciences, Seoul, Republic of Korea
}

\begin{abstract}
Background/Aim: Osteosarcoma is the most common type of bone cancer, but current therapeutic interventions remain largely insufficient. The development of new treatment strategies is needed, and moreover, optimal rodent models are necessary for testing the efficacy of new treatment modalities of osteosarcoma. Humanized mice carry human hematopoietic and immune systems, and are considered an ideal tool to study human diseases including cancer immunology. Herein, we performed a preliminary study toward developing an in vivo bioluminescent osteosarcoma model using humanized immunodeficient (NSG) mice. Materials and Methods: To establish the xenograft and orthotopic mouse model, NSG mice engrafted with human $\mathrm{CD}_{3} 4^{+}$hematopoietic stem cells were injected with luciferaseexpressing KHOS/NP cells at two different time points. Bioluminescence images were obtained to monitor in vivo tumor growth and metastasis. Influence of the degree of human cell engraftment on tumor growth and metastatic behavior was analyzed and compared between the two groups. Results: KHOS/NP-luc cells injected in humanized NSG mice formed macroscopic tumors. The percentage of human CD45+ cells in these models was similar, but the percentage of human CD45+CD3+ and their subset was higher in the late-injection group compared to that of the early-injection group. The rate of KHOS/NP tumor growth was higher in the early-injection
\end{abstract}

This article is freely accessible online.

*These Authors contributed equally to this study.

Correspondence to: Jun Ah Lee, MD, Ph.D., Department of Pediatrics, Center for Pediatric Cancer, National Cancer Center, 323 Ilsan-ro, Ilsandong-gu, Goyang, Gyeonggi-do 10408, Republic of Korea. Tel: +82 319201604, e-mail: junahlee@ncc.re.kr

Key Words: Osteosarcoma, humanized NSG mice, cell-derived xenograft, in vivo bioluminescence image. group than in the late-injection group. In the present study, human hematopoietic cell engraftment was not influenced by KHOS/NP cell injection, but KHOS/NP osteosarcoma showed more aggressive behavior in the early-injection group than that in the late-injection group, forming larger tumor volumes and earlier metastases. Conclusion: The results indicated that tumor growth and progression in humanized NSG mice may have been influenced by higher levels of human cell engraftment, especially $T$ cells. Although there exist some limitations to our study, our preliminary results can provide the basis for the development of a humanized osteosarcoma mouse model.

Osteosarcoma is the most common malignant bone tumor with a peak incidence occurring in adolescents. As the survival rate of patients with osteosarcoma has reached a plateau, the development of new treatment strategies based on its molecular characteristics are needed (1-3). As investigators search for novel agents, optimal in vitro or in vivo testing models are necessary. Animal models are widely used as preclinical platforms to test the efficacy of new treatment modalities. Due to the importance of bone and immune microenvironments in osteosarcoma, animal models recapitulating the human immune system are desirable in order to obtain more insight into the processes of osteosarcoma initiation, progression, and metastasis, as well as treatment sensitivity $(4,5)$. Super immunodeficient mice, such as NOD-scid IL2r $\gamma^{\text {null }}$ (NSG) mice, engraft human cells and tissues more efficiently than other models (6). Meanwhile, humanized mice carry human hematopoietic and immune systems and are considered an ideal tool to study hematopoiesis, infectious disease, and immunology (7-10).

Limited data exist on how to establish humanized mice bearing human tumors. Clearly, humanized NSG mice need specialized care and usually have a limited life span. In addition, the optimal time for tumor cell injection after humanization and the monitoring of tumor behavior in a less 
A

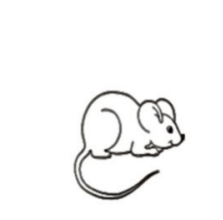

3-4 wks old NSG

Busulfan pre-conditioning at 48 and $24 \mathrm{~h}$ prior

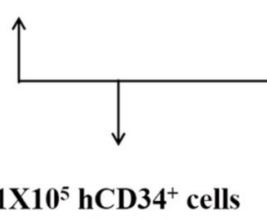
in retro orbital sinus $(O)$
Tumor size measurement

\& Bioluminescence imaging
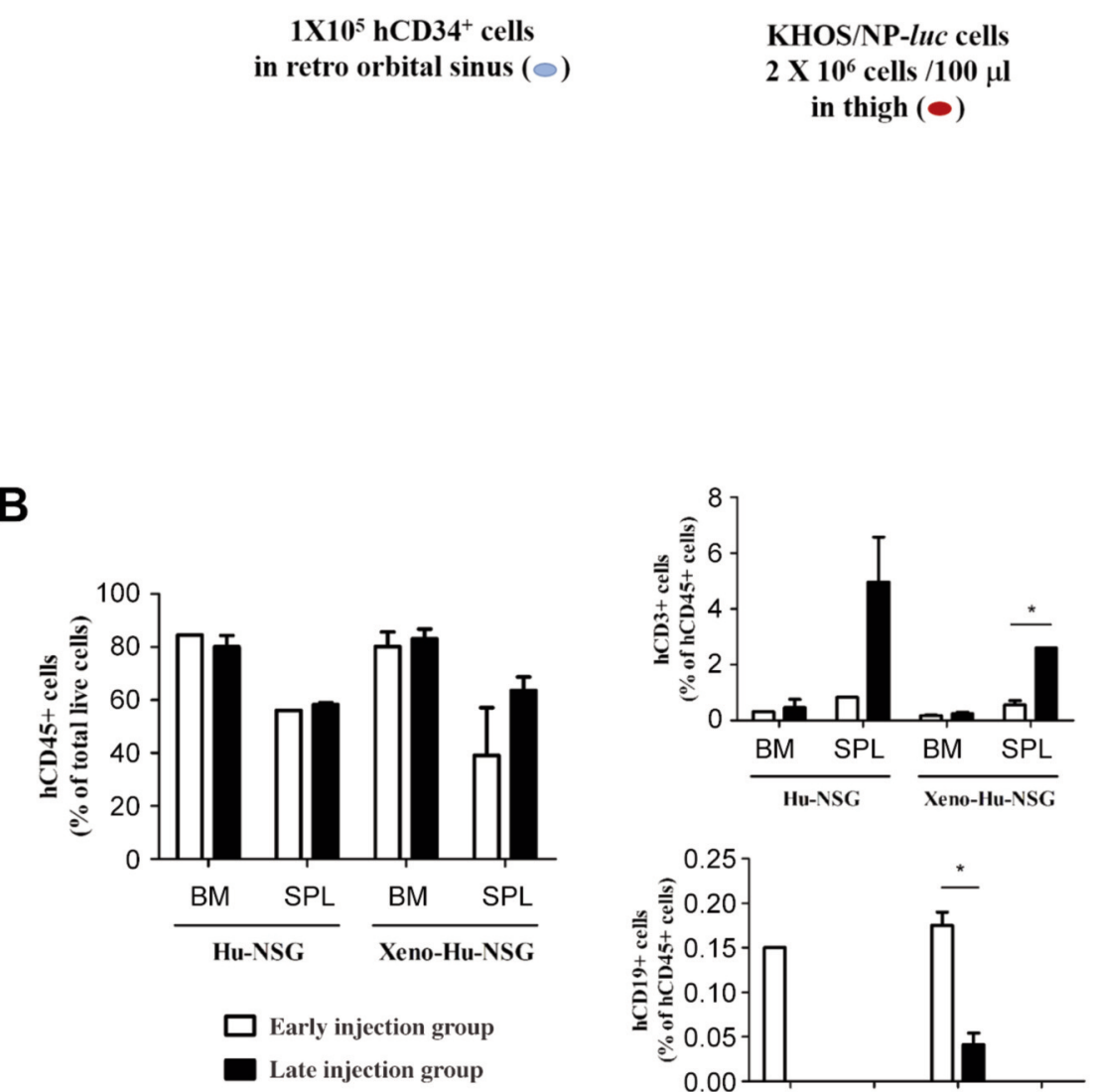
$8^{\text {th }}$ (early injection group)
or $17^{\text {th }}$ week (late injection group) or $21^{\text {th }}$ week (late injection group)

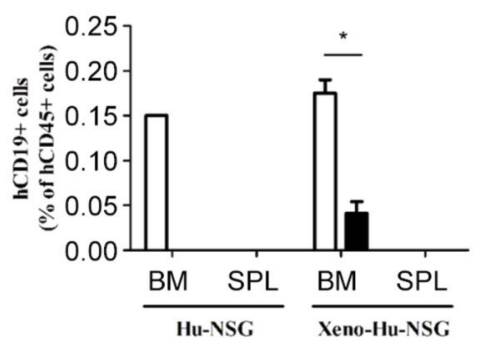

Sacrifice and Analysis

KHOS/NP-luc cells

$2 \times 10^{6}$ cells $/ 100 \mu \mathrm{l}$ in thigh $(\bullet)$

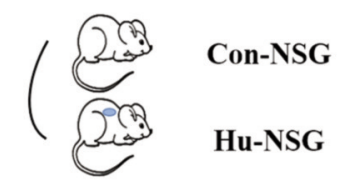

Con-NSG

Xeno-Hu-NSG

C Day after

tumor implantation

4

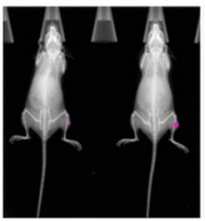

13

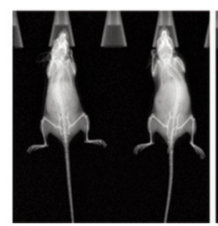

11

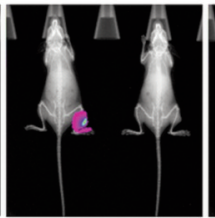

17

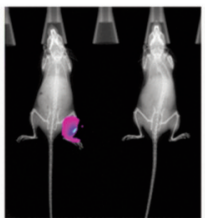

14

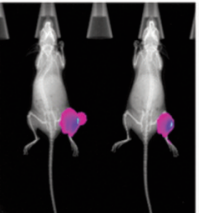

20

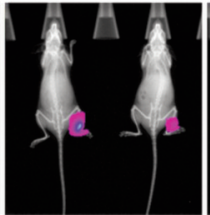

25
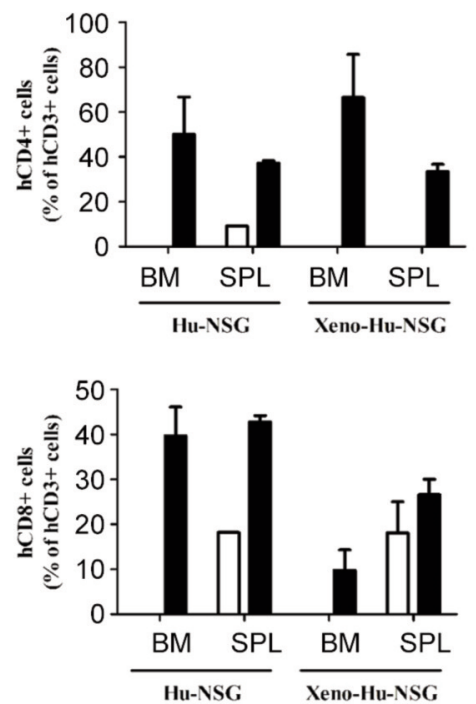

Late injection group

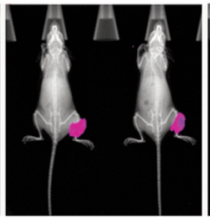

27

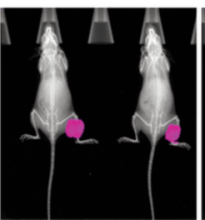

28

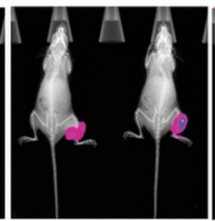

31

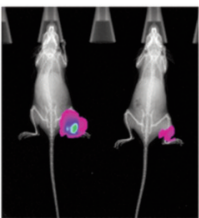

32

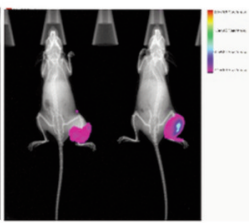

33

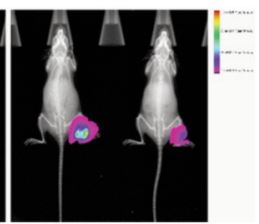

Figure 1. Continued 
D

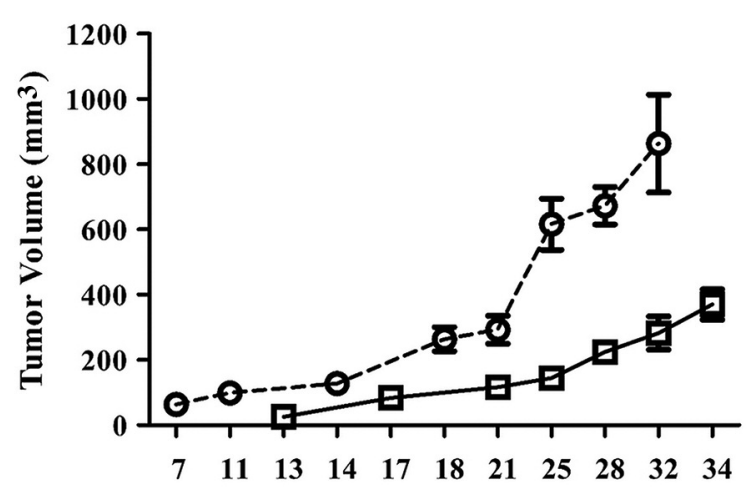

Day after tumor implantation

\section{- - Early injection group \# Late injection group}

Figure 1. Humanized osteosarcoma xenograft NSG model. (A) Experimental design and treatment schedule for generating Xeno-HuNSG mice. (B) Flow cytometric analysis of NSG mice transplanted with human CD34+ cells and KHOS/NP-luc cells. Bone marrow and spleens were collected from the mice, single-cell preparations were stained, and the human cell reconstitution was analyzed at different times. The percentages are represented as the mean $\pm S E M .(C, D)$ In vivo imaging and tumor measurements of Xeno-Hu-NSG mice. After KHOS/NP-luc cell injection, mice were imaged using a Bruker In-Vivo Xtreme system and the images quantified as photons/second using Bruker's software. Tumor volumes were calculated on the indicated days. Details are described in Materials and Methods. Con-NSG: Normal NSG mice, HuNSG: humanized NSG mice, Xeno-Hu-NSG: humanized osteosarcoma xenograft NSG mice, BM: bone marrow, SPL: spleen, ${ }^{*} p<0.05$.

invasive manner are matters of debate. We previously observed a time-dependent increase of human cell engraftment in NSG mice following human $\mathrm{CD}^{+} 4^{+}$cell transplantation with busulfan conditioning $(11,12)$. Considering the interaction between engrafted human immune cells and tumor cells in NSG mice, we presume the timing of tumor cell injection after humanization may influence tumor behavior. In the current study, we aimed to establish a humanized osteosarcoma model using NSG mice. The humanized NSG mice were injected with luciferaseexpressing KHOS/NP cells at two different time points after human $\mathrm{CD}_{3} 4^{+}$cell transplantation ( 8 weeks and 17 weeks), for both a xenograft and an orthotopic model. Tumor growth and metastatic behavior was then evaluated and compared using in-vivo bioluminescence images.

\section{Materials and Methods}

Osteosarcoma cells. The human osteosarcoma cell line KHOS/NP was purchased from the American Type Culture Collection (ATCC; Manassas, VA, USA). The cells were maintained in Minimum
Essential Medium $\alpha$ (MEM $\alpha$; Thermo Fisher Scientific, Inc., Waltham, MA, USA) supplemented with $10 \%$ fetal bovine serum (Thermo Fisher Scientific, Inc.), under $5 \% \mathrm{CO}_{2}$ conditions at $37^{\circ} \mathrm{C}$.

Transfection with luciferase. The KHOS/NP cells were transfected with a luciferase expression vector (Promega, Madison, WI, USA) and selected under culture conditions including hygromycin B (Thermo Fisher Scientific, Inc.) according to the manufacturer's instructions. Luciferase-expressing KHOS/NP osteosarcoma cells (KHOS/NP-luc cells) were tested for promotor activation using a luciferase assay (Thermo Fisher Scientific, Inc.) and a Bruker InVivo Xtreme imaging system (Bruker BioSpin Corp., Billerica, MA, USA).

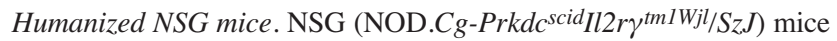
were purchased from the Jackson Laboratory (Bar Harbor, ME, USA). For all experiments, mice were bred as a homozygous line and maintained under specific pathogen-free conditions at the Laboratory of Animal Research Center, Korea Institute of Radiological Medical Sciences (Seoul, Republic of Korea). Experiments were conducted according to the guidelines for ethical use of animals under an approved protocol (KIRAMS 2017-0046). Twelve 3-4-week-old female NSG mice were randomly divided into three groups (control, early-injection group, late-injection group) and conditioned with busulfan (Korea Otsuka Pharmaceutical, Seoul, Republic of Korea). Busulfan was dissolved in dimethyl sulfoxide (DMSO; Sigma-Aldrich Corporation, St. Louis, MO, USA), diluted with $0.9 \%$ saline, and intraperitoneally injected into the NSG mice ( $25 \mathrm{mg} / \mathrm{kg}$ body weight, $500-625 \mu \mathrm{g}$ per dose) 48 and $24 \mathrm{~h}$ prior to transplantation. To generate humanized NSG (Hu-NSG) mice, each mouse was intravenously transplanted via the retro-orbital sinus with $1 \times 10^{5}$ human CD34+ cells (hCD34+; Thermo Fisher Scientific, Inc.) in $100 \mu \mathrm{l}$ phosphate-buffered saline (PBS). Enofloxacin $(0.27 \mathrm{mg} / \mathrm{ml})$ was used as a prophylactic antibiotic in the drinking water of the mice to prevent urinary tract infections.

Xenograft and orthotopic mice model. For the xenograft (Xeno-HuNSG) model (Figure 1A), $2 \times 10^{6} \mathrm{KHOS} / \mathrm{NP}-l$ luc cells were injected subcutaneously into the thighs of Hu-NSG mice under $3 \%$ isoflurane-induced general anesthesia on the $8^{\text {th }}$ or $17^{\text {th }}$ week post hCD34+ cell transplantation. Tumor volumes were then measured with precision calipers every 3-4 days for 4 weeks. For the orthotopic (Ortho-Hu-NSG) model (Figure 2A), $1 \times 10^{5}$ KHOS/NP$l u c$ cells were injected into knee joints of Hu-NSG mice under $3 \%$ isoflurane-induced general anesthesia on the $8^{\text {th }}$ or $17^{\text {th }}$ week post hCD34+ cell transplantation and monitored for 2-4 weeks based on physical conditions of the mice. The general health of the transplanted mice was monitored daily.

In vivo bioluminescence images. All the mice under 3\% isoflurane anesthesia were intraperitoneally injected twice a week with 150 $\mathrm{mg} / \mathrm{kg}$ D-luciferin (Thermo Fisher Scientific, Inc.), and then imaged using the Bruker In-Vivo Xtreme imaging system.

Analysis after KHOS/NP-luc cell injection. For analysis of human cell engraftment, the mice were sacrificed 2-4 weeks after KHOS/NP cell injection. Mononuclear cells were isolated from the bone marrow and spleens of the mice and single-cell suspensions were prepared using standard procedures. The cells were then stained with the following antibodies: hCD45-APC (Miltenyi 
A

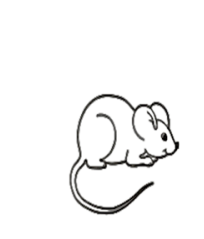

3-4 wks old NSG

Busulfan pre-conditioning at 48 and $24 \mathrm{~h}$ prior

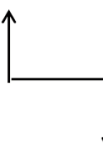

$1 \mathrm{X10}^{5} \mathrm{hCD} 34+$ cells in retro orbital sinus (O)

\section{Bioluminescence imaging}

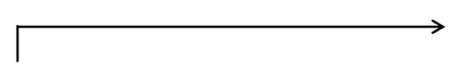

\begin{abstract}
$8^{\text {th }}$ (early injection group) or $17^{\text {th }}$ week (late injection group)

$10.5^{\text {th }}$ week (early injection group) or $21^{\text {th }}$ week (late injection group)
\end{abstract}
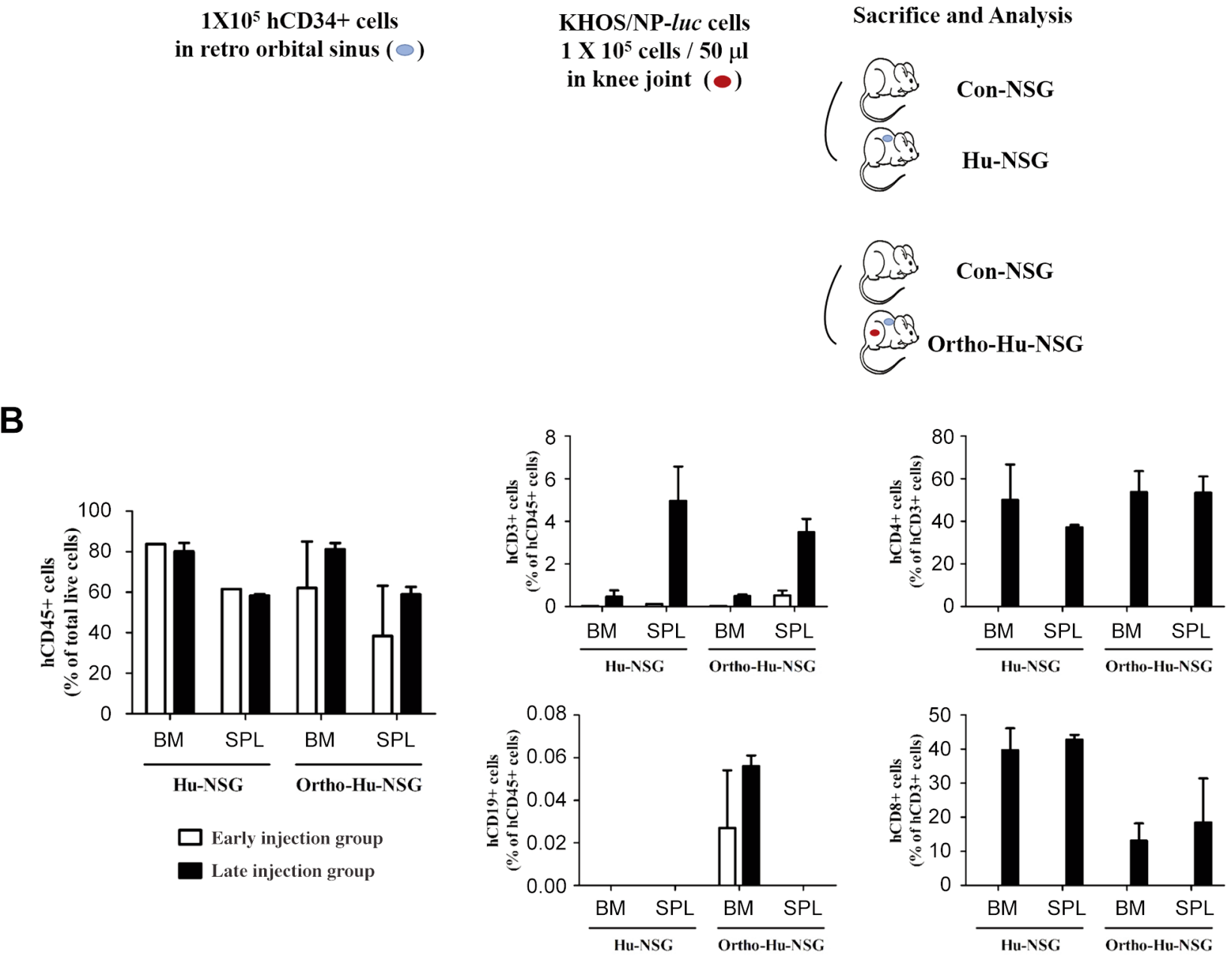

Figure 2. Continued

Biotec, Madrid, Spain), and hCD3-FITC, hCD19- PE, hCD4-PE, and hCD8-PE-Cy5 (BD Biosciences, San Jose, CA, USA). Flow cytometry was performed using a FACSCanto ${ }^{\mathrm{TM}}$ II Cell Analyzer (BD Biosciences) with ten thousand to one million events acquired per sample and then analyzed using FACSDiva and Flowjo software (BD Biosciences).

Statistical analysis. All statistical analyses were performed using GraphPad Prism 5.0 (GraphPad Software, Inc., San Diego, CA, USA). Quantitative variables are expressed as mean \pm standard deviation (SD) and differences between groups were considered to be statistically significant at a $p$-value $<0.05$.

\section{Results}

KHOS/NP-luc cells were tested after selection using hygromacin B resistance to confirm luciferase expression (data not shown). Hu-NSG mice were humanized as previously described and then injected with KHOS/NP-luc 
C

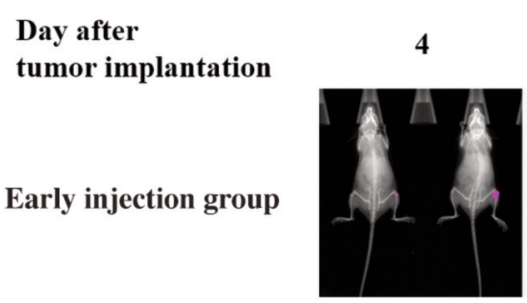

13

Late injection group

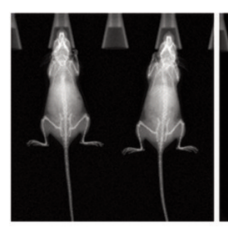

7

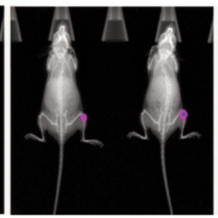

17

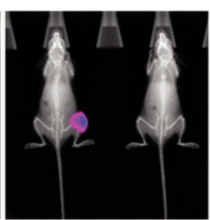

11

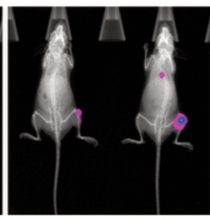

20

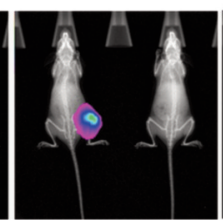

14

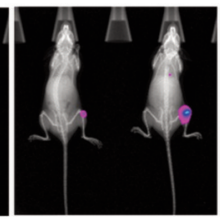

27

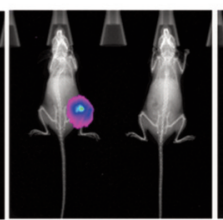

18

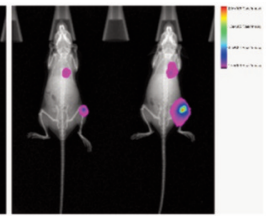

31

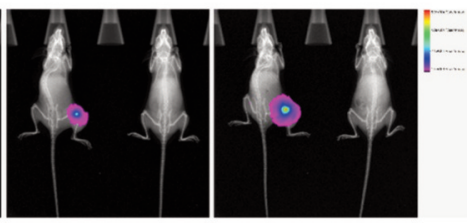

Figure 2. Humanized osteosarcoma orthotopic NSG model. (A) Experimental design and treatment schedule for generating Ortho-Hu-NSG mice. (B) Flow cytometric analysis of NSG mice transplanted with human CD34+cells and KHOS/NP-luc cells. Bone marrow and spleen were collected from the mice, single-cell preparations stained, and the human cell reconstitution analyzed at different times. The percentages are represented as the mean $\pm S E M .(C)$ In vivo imaging of Ortho-Hu-NSG mice. After KHOS/NP-luc cell injection, mice were imaged using a Bruker In-Vivo Xtreme system and the images quantified as photons/second using Bruker's software. Details are described in Materials and Methods. Con-NSG: Normal NSG mice, Hu-NSG: humanized NSG mice, Xeno-Hu-NSG: humanized osteosarcoma xenograft NSG mice, BM: bone marrow, SPL: spleen.

cells at the $8^{\text {th }}$ week (early-injection group) or $17^{\text {th }}$ week (late-injection group) after transplantation with human $\mathrm{CD}^{+} 4^{+}$cells as Xeno-Hu-NSG and Ortho-Hu-NSG osteosarcoma models.

Mice of the humanized osteosarcoma xenograft model were sacrificed 4 weeks after KHOS/NP-luc cell injection and analyzed for human cell engraftment by flow cytometry (Figure 1B). The percentage of human $\mathrm{CD} 45^{+}$cells in bone marrow of Xeno-Hu-NSG mice were $80.2 \%$ in the earlyinjection group and $83.1 \%$ in the late-injection group, similar to that of the control Hu-NSG mice. The percentage of human $\mathrm{CD}_{4} 5^{+}$cells tended to be higher in spleen of Xeno$\mathrm{Hu}-\mathrm{NSG}$ mice in the late-injection group than that in the early-injection group (63.6\% vs. $39.15 \%, p=0.2)$. Human $\mathrm{CD} 45^{+} \mathrm{CD} 19^{+}$cells were detected in only the bone marrow and the engraftment rate was higher in the Xeno-Hu-NSG early-injection group compared to that of the late-injection group $(0.18 \%$ vs. $0.04 \%, p=0.047)$. The percentage of human $\mathrm{CD}_{4} 5^{+} \mathrm{CD} 3^{+}$cells of bone marrow tended to be higher in the late-injection group than the early-injection group $(0.24 \% \mathrm{vs}$. $0.18 \%, p=0.27$ ). However, human $\mathrm{CD} 45^{+} \mathrm{CD}^{+}$in spleen was significantly higher in the late-injection group compared to that of the early-injection group $(0.56 \%$ vs. $2.6 \%, p=0.02)$. When T-cell subsets were analyzed among the gated human $\mathrm{CD} 3+$ population, $\mathrm{CD} 3{ }^{+} \mathrm{CD} 4^{+}$cells were detected in only the late-injection group and found to be $66.45 \%$ of bone marrow and $31.8 \%$ of spleen. The percentages of $\mathrm{CD} 3^{+} \mathrm{CD} 8^{+}$cells tended to be higher in the late-injection group compared to the early-injection group, being $9.78 \%$ vs. $0 \%$, respectively, in bone marrow $(p=0.14)$ and $26.55 \%$ vs. $18.05 \%$, respectively, in spleen $(p=0.22)$ KHOS/NP-luc cells injected in NSG mice formed macroscopic tumors, but luciferasederived signals decreased in a time-dependent manner. The rate of KHOS/NP tumor growth was higher in the earlyinjection group than that in the late-injection group with a 2.3-fold larger tumor volume at the end of the experiment (Figure 1C and D).

Mice of the early-injection group of the humanized osteosarcoma orthotopic model were sacrificed on the $18^{\text {th }}$ day post KHOS/NP-luc cell injection due to the deteriorating condition of the Ortho-Hu-NSG mice (following IACUC guidelines), while mice of the late-injection group were sacrificed on the $4^{\text {th }}$ week post KHOS/NP-luc cells injection (Figure 2B). The engraftment rate of human $\mathrm{CD} 45^{+}$cells was higher in bone marrow of the late-injection group compared to that of the early-injection group $(81.2 \%$ vs. $62.1 \%$, respectively), as well as in the spleen $(58.85 \%$ vs. $38.4 \%$, respectively). Human $\mathrm{CD} 45^{+} \mathrm{CD} 19^{+}$cells were detected only in the bone marrow and exhibited slightly higher engraftment rates in the late-injection group than that in the early-injection group $(0.056 \%$ vs. $0.027 \%, p=0.24)$. The percentage of human $\mathrm{CD}_{4} 5^{+} \mathrm{CD}^{+}$cells was slightly higher in the lateinjection group than that of the early-injection group in bone marrow $(0.49 \%$ vs. $0.01 \%, p=0.05)$ and in spleen $(3.49 \%$ vs. $0.53 \%, p=0.07)$. Human T-cell subsets were detected in the bone marrow and spleen of only the late-injection group. In bone marrow, the percentages of $\mathrm{CD}^{+} \mathrm{CD}^{+}$and $\mathrm{CD} 3{ }^{+} \mathrm{CD} 8^{+}$ cells were $58.3 \%$ and $13.1 \%$, respectively. In the spleen, 
percentages of $\mathrm{CD}^{+}{ }^{+} \mathrm{CD} 4^{+}$and $\mathrm{CD}^{+}{ }^{+} \mathrm{CD} 8^{+}$cells were $53.4 \%$ and $18.48 \%$, respectively. The luciferase signal decreased in a time-dependent manner in the Ortho-Hu-NSG mice. Mice of the early-injection group tended to develop metastasis earlier and demonstrated a faster rate of tumor growth (Figure $2 \mathrm{C})$. For mice of the early-injection group, luciferase signals were detected in the knee on day 4 and in the lungs on day 11. For mice of the late-injection group, luciferase signals were detected on day 13 in the knee. Although luciferase signals were not detected in areas of the chest, autopsy revealed multiple metastatic nodules in lungs.

\section{Discussion}

Animal models are useful for testing the efficacy of new treatment modalities of human diseases. Various approaches have been attempted to overcome the genetic differences between humans and animals, including the use of humanized mice. In a previous study, we observed that NSG mice support high levels of engraftment following injection with human $\mathrm{CD} 4^{+}$cells. Herein, we performed a preliminary study to develop an in-vivo bioluminescent osteosarcoma model in humanized NSG mice.

Human hematopoietic cell engraftment in the current study was not influenced by KHOS/NP cell injection. The fractions of human $\mathrm{CD} 45^{+}$cells in model mice were similar to those of the control Hu-NSG mice, irrespective of the KHOS/NP transplanted sites and time points analyzed. Although the same number of KHOS/NP cells were transplanted, KHOS/NP osteosarcoma showed a more aggressive behavior in the early-injection group than that in the late-injection group, forming larger tumor volume and earlier metastases. We presume the cause may have been the interaction between the KHOS/NP cells and human hematopoietic cells or immune cells that replaced the endogenous mouse hematopoietic system. Tumor growth and progression in NSG mice may have been influenced by the degree of human cell engraftment, especially T cells. In our previous work, both B-cell and T-cell engraftment increased in a time-dependent manner after human hematopoietic stem cell transplantation. We observed that B-cell engraftment rates were similar between early-injection and late-injection groups. However, the percentages of human $\mathrm{T}$ cells (both $\mathrm{CD}^{+} \mathrm{CD}^{+}$and $\mathrm{CD}^{+} \mathrm{CD}^{+}$) were higher in mice of the lateinjection group. $\mathrm{T}$ cells in the tumor microenvironment play an essential role in immune surveillance (13-15). High level of T-cell infiltration in tumors is associated with better prognosis and response in several types of cancer (16-19). In the current study, we did not analyze the $\mathrm{T}$ cells in the KHOS/NP tumors formed in the NSG mice. As NSG mice are constitutively lacking $\mathrm{T}$ cells, $\mathrm{T}$ cells in tumor microenvironment would derive from engrafted $\mathrm{T}$ cells. Number of T cells in the KHOS/NP tumor microenvironment might correlate with T cell engraftment. Accordingly, we surmise that there might have been more interaction between $\mathrm{T}$ cells and KHOS/NP tumor cells in the late injection group than in the early injection group, and inhibited KHOS/NP tumor progression.

According to the Jackson laboratory's report, C57BL/6J mice of approximately 3 or 4 weeks of age corresponds to 12.5 years of age in humans. Accordingly, 8 weeks (early injection) and 17 weeks (late injection) would correspond to 20 and 30 years of age, respectively. Considering the chronologic factors, mice of the early-injection group would correspond to osteosarcoma of adolescents and those of the late-injection group would correspond to human adults. The clinical characteristics and prognosis of osteosarcoma in humans differ according to age, with worse prognosis for adults. In the current study, we observed the opposite finding, with KHOS/NP tumors in the early-injection group showing a more aggressive behavior.

Our study had several limitations due to its preliminary nature. For instance, the number of mice tested was small, which may have affected the ability to reach statistical significance for some of the end-points evaluated. We failed to observe lung metastasis in Ortho-Hu-NSG mice of the late-injection group using the Bruker In-Vivo Xtreme imaging due to decreasing luciferase expression in the KHOS/NP cells, which occurred in a time-dependent manner. Therefore, establishing a technique or condition for stable luciferaseexpression in KHOS/NP cells is needed.

In conclusion, human hematopoietic cell engraftment was not influenced by KHOS/NP cell injection in the examined models. KHOS/NP osteosarcoma showed more aggressive behavior in the early-injection group than that in the lateinjection group, forming larger tumor volume and earlier metastases. The results indicated that tumor growth and progression in the humanized NSG mice may have been influenced by higher levels of human cell engraftment, especially $\mathrm{T}$ cells. Taken together, our preliminary results provide a basis for the development of humanized osteosarcoma mice models. Further studies are necessary to establish more sophisticated immunodeficient mice with greater human T-cell engraftment, as well as develop more sensitive in vivo tumor imaging techniques.

\section{Conflicts of Interest}

The Authors declare that there are no conflicts of interest.

\section{Authors' Contributions}

Yunmi Ko: Project administration, methodology, experiment, visualization, writing of original draft; Yeon Ho Jeong; methodology, validation, data curation; Jin-Hee Seo: Supporting in animal experiments; Jun Ah Lee: Supervision, conceptualization, data curation, writing review and editing. 


\section{Acknowledgements}

This research was supported by the Basic Science Research Program through the National Research Foundation of Korea (NRF) funded by the Ministry of Education (NRF-2017R1D1A1B03036574) and 2016 Research Grant from Kangwon National University (No. 520160190).

\section{References}

1 Wedekind MF, Wagner LM and Cripe TP: Immunotherapy for osteosarcoma: Where do we go from here? Pediatr Blood Cancer 65(9): e27227, 2018. PMID: 29923370. DOI: 10.1002/pbc.27227

2 Luetke A, Meyers PA, Lewis I and Juergens H: Osteosarcoma treatment - where do we stand? A state of the art review. Cancer Treat Rev 40(4): 523-532, 2014. PMID: 24345772. DOI: 10.1016/j.ctrv.2013.11.006

3 Hong W, Yuan H, Gu Y, Liu M, Ji Y, Huang Z, Yang J and Ma L: Immune-related prognosis biomarkers associated with osteosarcoma microenvironment. Cancer Cell Int 20: 83, 2020. PMID: 32190007. DOI: 10.1186/s12935-020-1165-7

4 Koirala P, Roth ME, Gill J, Piperdi S, Chinai JM, Geller DS, Hoang BH, Park A, Fremed MA, Zang X and Gorlick R: Immune infiltration and PD-L1 expression in the tumor microenvironment are prognostic in osteosarcoma. Sci Rep 6: 30093, 2016. PMID: 27456063. DOI: 10.1038/srep30093

5 Morillon YM 2nd, Sabzevari A, Schlom J and Greiner JW: The development of next-generation PBMC humanized mice for preclinical investigation of cancer immunotherapeutic agents Anticancer Res 40(10): 5329-5341, 2020. PMID: 32988851. DOI: 10.21873/anticanres.14540

6 Walsh NC, Kenney LL, Jangalwe S, Aryee KE, Greiner DL, Brehm MA and Shultz LD: Humanized mouse models of clinical disease. Annu Rev Pathol 12: 187-215, 2017. PMID: 27959627. DOI: 10.1146/annurev-pathol-052016-100332

7 Victor Garcia J: Humanized mice for HIV and AIDS research. Curr Opin Virol 19: 56-64, 2016. PMID: 27447446. DOI: 10.1016/j.coviro.2016.06.010

8 Shultz LD, Brehm MA, Garcia-Martinez JV and Greiner DL: Humanized mice for immune system investigation: progress, promise and challenges. Nat Rev Immunol 12(11): 786-798, 2012. PMID: 23059428. DOI: 10.1038/nri3311

9 Agarwal Y, Beatty C, Ho S, Thurlow L, Das A, Kelly S, Castronova I, Salunke R, Biradar S, Yeshi T, Richardson A and Bility M: Development of humanized mouse and rat models with full-thickness human skin and autologous immune cells. Sci Rep 10(1): 14598, 2020. PMID: 32884084. DOI: 10.1038/s41598020-71548-Z

$10 \mathrm{Ko}$ Y, Jeong YH and Lee JA: Transplantation of human umbilical cord blood $\mathrm{CD} 4^{+}$cells into the liver of newborn NOD/SCID/IL-2R $\gamma$ null (NSG) mice after busulfan conditioning. Blood Res 52(4): 316-319, 2017. PMID: 29333410. DOI: $10.5045 /$ br.2017.52.4.316

11 Kang YK, Ko Y, Choi A, Choi HJ, Seo JH, Lee M and Lee JA: Humanizing NOD/SCID/IL-2R $\gamma$ null (NSG) mice using busulfan and retro-orbital injection of umbilical cord blood-derived CD34(+) cells. Blood Res 51(1): 31-36, 2016. PMID: 27104189. DOI: $10.5045 / \mathrm{br} .2016 .51 .1 .31$

12 Ashizawa $\mathrm{T}$, Iizuka A, Nonomura $\mathrm{C}$, Kondou R, Maeda C, Miyata H, Sugino T, Mitsuya K, Hayashi N, Nakasu Y, Maruyama K, Yamaguchi K, Katano I, Ito M and Akiyama Y:
Antitumor effect of programmed death-1 (PD-1) blockade in humanized the NOG-MHC double knockout mouse. Clin Cancer Res 23(1): 149-158, 2017. PMID: 27458246. DOI: 10.1158/ 1078-0432.CCR-16-0122

13 Morton CL and Houghton PJ: Establishment of human tumor xenografts in immunodeficient mice. Nat Protoc 2(2): 247-250, 2007. PMID: 17406581. DOI: 10.1038/nprot.2007.25

14 Wang Z, Li B, Ren Y and Ye Z: T-cell-based immunotherapy for osteosarcoma: challenges and opportunities. Front Immunol 7: 353, 2016. PMID: 27683579. DOI: 10.3389/fimmu.2016.00353

15 Chung YS, Son JK, Choi B, Joo SY, Lee YS, Park JB, Moon H, Kim TJ, Kim SH, Hong S, Chang J, Kang MS and Kim SJ: Cotransplantation of human fetal thymus, bone and CD34(+) cells into young adult immunodeficient NOD/SCID IL2R $\gamma$ (null) mice optimizes humanized mice that mount adaptive antibody responses. Clin Immunol 157(2): 156-165, 2015. PMID: 25725428. DOI: 10.1016/j.clim.2015.02.005

16 Halkias J, Yen B, Taylor KT, Reinhartz O, Winoto A, Robey EA and Melichar HJ: Conserved and divergent aspects of human Tcell development and migration in humanized mice. Immunol Cell Biol 93(8): 716-726, 2015. PMID: 25744551. DOI: 10.1038/icb.2015.38

17 Knibbe-Hollinger JS, Fields NR, Chaudoin TR, Epstein AA, Makarov E, Akhter SP, Gorantla S, Bonasera SJ, Gendelman HE and Poluektova LY: Influence of age, irradiation and humanization on NSG mouse phenotypes. Biol Open 4(10): 1243-1252, 2015. PMID: 26353862. DOI: 10.1242/bio.013201

18 Rongvaux A, Willinger T, Martinek J, Strowig T, Gearty SV, Teichmann LL, Saito Y, Marches F, Halene S, Palucka AK, Manz MG and Flavell RA: Development and function of human innate immune cells in a humanized mouse model. Nat Biotechnol 32(4): 364-372, 2014. PMID: 24633240. DOI: 10.1038/nbt.2858

19 Brehm MA, Racki WJ, Leif J, Burzenski L, Hosur V, Wetmore A, Gott B, Herlihy M, Ignotz R, Dunn R, Shultz LD and Greiner DL: Engraftment of human HSCs in nonirradiated newborn NOD-scid IL2r $\gamma$ null mice is enhanced by transgenic expression of membrane-bound human SCF. Blood 119(12): 2778-2788, 2012. PMID: 22246028. DOI: 10.1182/blood-2011-05-353243

20 Brehm MA, Shultz LD, Luban J and Greiner DL: Overcoming current limitations in humanized mouse research. J Infect Dis 208 Suppl 2: S125-S130, 2013. PMID: 24151318. DOI: 10.1093/infdis/jit319

21 Covassin L, Jangalwe S, Jouvet N, Laning J, Burzenski L, Shultz LD and Brehm MA: Human immune system development and survival of non-obese diabetic (NOD)-scid IL2r $\gamma($ null) (NSG) mice engrafted with human thymus and autologous haematopoietic stem cells. Clin Exp Immunol 174(3): 372-388, 2013. PMID: 23869841. DOI: $10.1111 /$ cei.12180

22 Ito R, Takahashi T, Katano I and Ito M: Current advances in humanized mouse models. Cell Mol Immunol 9(3): 208-214, 2012. PMID: 22327211. DOI: $10.1038 / \mathrm{cmi} .2012 .2$

23 Uluçkan Ö, Segaliny A, Botter S, Santiago JM and Mutsaers AJ: Preclinical mouse models of osteosarcoma. Bonekey Rep 4: 670, 2015. PMID: 25987985. DOI: 10.1038/bonekey.2015.37

Received March 11, 2021

Revised April 23, 2021

Accepted April 26, 2021 